rev Psi

Revista de Psicología (UNLP)

https://revistas.unlp.edu.ar/revpsi

\title{
Variétés de la lettre et objets autistiques
}

\author{
Éric Laurent ${ }^{1,2}$ \\ Adresse électronique \\ ericlaurent@lacanian.net \\ Traduction et correction d'épreuves \\ Traduction: Luis H. Volta | Correction dépreuves: Nicolás Alessandroni, María Cristina Piro
}

Affiliations institutionnelles

${ }^{1}$ Association Mondiale de Psychanalyse (AMP) $\quad{ }^{2}$ Université Paris 8 Vincennes - Sant-Denis (France)

\section{Résumé}

Pour répondre à la variété de sujets relevant du spectre autistique et à la diversité des lieux qui les accueillent et sont attentifs au bain de langage dans lequel ces sujets sont plongés, l'orientation de notre travail vise à définir la position d'un thérapeute partenaire respectueux des multiples aspects que peut prendre l'effet mutatif d'une rencontre nouvelle avec la langue. Dans cette perspective, plutôt que la recherche d'une méthode idéale d’approche du sujet avec autisme, il s'agit de présenter ici la diversité de résultats obtenus dans des contextes les plus variés, avec le trait constant de soutenir l'effort d'inscription du sujet dans toutes les dimensions de la langue, de l'instance de la lettre et du traitement des objets hors corps que sont le regard et la voix, dans la plus grande variété de situations ou d'ateliers possibles, tout en maintenant des apprentissages. (extrait du manuscrit).

\section{Mots clés}

autisme | psychanalyse | variétés de la lettre | objets autistiques

\section{Processus éditorial}

Reçu

4 may. 2021

Accepté

23 jun. 2021 10.24215/2422572XE114 objets autistiques. Revista de Psicología
1 ère décision

23 jun. 2021

Publié

31 jul. 2021
Responsable du dossier

María Cristina Piro (Faculté de Psychologie UNLP, Argentine)
ISSN

2422-572X

Licence

Licence Culture Libre CC-BY 4.0

(Partager - Adapter - Attribuer)

Entité de publication

RevPsi est une publication de la

Faculté de Psychologie (Universidad

Nacional de La Plata, Argentine) 


\section{Variedades da letra e objetos autisticos}

\section{Resumo}

A fim de responder à variedade de sujeitos dentro do espectro autista e à diversidade de lugares que os recebem e prestam atenção ao banho de língua em que esses sujeitos estão imersos, a orientação de nosso trabalho visa definir a posição de um terapeuta que seja um partenaire respeitoso dos múltiplos aspectos que o efeito transformador de um novo encontro com a língua pode tomar. Nesta perspectiva, em vez de buscar um método ideal para a abordagem do sujeito autista, o objetivo é apresentar aqui a diversidade de resultados obtidos nos mais variados contextos, cuja característica constante é sustentar o esforço de inscrever o sujeito em todas as dimensões da linguagem, da instância da letra e do tratamento de objetos fora do corpo como o olhar e a voz, na grande variedade de situações ou oficinas possíveis, mantendo o processo de aprendizagem (fragmento do manuscrito).

\section{Palavras-chave}

autismo | psicanálise | variedades da letra | objetos autisticos

\section{Varieties of the letter and autistic objects}

\section{Abstract}

In order to respond to the variety of subjects within the autistic spectrum and the diversity of settings that receive them and pay attention to the language bath in which these subjects are immersed, our work aims at defining the position of a partenaire therapist who respects the multiple aspects that the transforming effect of a new encounter with language can take. From this perspective, rather than searching for an ideal method for the approach of the autistic subject, we present a diversity of results obtained in varied contexts whose constant feature is to sustain the effort of inscribing the subject in all the dimensions of language, the instance of the letter and the treatment of objects outside the body -such as the gaze and the voice-, in a great variety of situations and workshops, while maintaining the learning process (fragment of the manuscript).

\section{Keywords}

autism | psychoanalysis | varieties of the letter | autistic objects 
Pour répondre à la variété de sujets relevant du spectre autistique et à la diversité des lieux qui les accueillent et sont attentifs au bain de langage dans lequel ces sujets sont plongés, lorientation de notre travail vise à définir la position d'un thérapeute partenaire respectueux des multiples aspects que peut prendre l'effet mutatif d'une rencontre nouvelle avec la langue.

Dans cette perspective, plutôt que la recherche d'une méthode idéale d'approche du sujet avec autisme, il s'agit de présenter ici la diversité de résultats obtenus dans des contextes les plus variés, avec le trait constant de soutenir l'effort d'inscription du sujet dans toutes les dimensions de la langue, de l'instance de la lettre et du traitement des objets hors corps que sont le regard et la voix, dans la plus grande variété de situations ou d'ateliers possibles, tout en maintenant des apprentissages.

La série des machines sur lesquelles se sont appuyés des sujets autistes pour construire leur monde est très large. Elle comprend l’appareil photographique dont s'est servi Garance pour construire son rapport à «l'espace qui ne se voit pas» (Reliquet y Reliquet, 2011, p. 98; Laurent, 2012, p. 83), l'ordinateur qui a servi à Birger Sellin pour sa communication assistée, le logiciel de reconnaissance de voix Siri pour Gus (Newman, 2014), le jeu Minecraft pour beaucoup. Vont bientôt s'y ajouter une adaptation des lunettes Google munies d'un logiciel de reconnaissance des visages et des émotions, mises au point par un chercheur de Stanford -au départ pour son cousin autiste qui sessayait à la reconnaissance démotions devant son miroir (Metz, 2019)-, ainsi que divers robots néo-humains ou néo-animaux (Chérel, 2019).

Pour autant, il ne s'agit pas de réduire ces sujets à des «enfants machines» et de croire qu'ils seraient de purs ordinateurs. Ces sujets ont un corps, et c'est ce corps qu'ils ont qui se trouve plongé dans un bain de langage. C'est le langage qui se trouve être la première machine rencontrée par ces corps et dans lequel le vivant doit trouver à se loger. Avec sa synesthésie, Daniel Tammet est bien placé pour nous dire que ce qu'il expérimente quand il calcule, aucune machine de Turing ne pourrait l'appréhender. Il faut s'intéresser à l'utilisation des machines comme «assistants du sujet», sans pour autant réduire le sujet-corps à une machine. C’est ce que permet la considération de la rencontre avec le langage, qui laisse des traces «traumatiques» pour tout sujet qui naît au langage, que le sujet parle ou non. C’est le véritable «traumatisme de la naissance», et il prend pour le sujet autiste des formes particulières. Les machines mettent spécialement bien en valeur la multiplicité des registres que nous nommons la lettre, autre nom de ce qui fonctionne comme un à travers ce que nous appelons parler, écrire, compter ou se représenter une image.

\section{Variétés de la lettre. L'exemple de l'Affinity Therapy}

Partons du témoignage des efforts de la famille de Ron Suskind pour traiter l'autisme de leur enfant, publié sous le titre Life: Animated (Suskind, 2016¹). Le récit de Cornelia et Ron Suskind est fascinant en tant qu'expérience d'une bataille inventive de toute la famille pour inventer une langue et une méthode à partir d'un intérêt 
spécifique de l'enfant. Ce livre a trouvé un écho qui a donné lieu à nombre d'entretiens et a résonné pour de nombreuses personnes. Je retiendrai quelques moments clefs. Tout d'abord, la première interprétation de Cornelia au milieu des années 1990, alors qu'ils venaient d’emménager à Washington DC où Ron avait été embauché comme journaliste chargé de la politique intérieure (National Affairs) au Wall Street Journal.

Simplement timide jusquà son troisième anniversaire, Owen a développé une forme d'autisme régressif, ce qui signifie qu'il a essentiellement arrêté de communiquer. Une chose qu'il aimait toujours faire était de regarder les dessins animés Disney avec son frère, et il regardait les mêmes scènes encore et encore, encore [...] Puis un jour, en regardant La petite sirène, Owen a dit son premier mot depuis longtemps : “juicervose”. Sa mère, Cornelia, a compris qu’il était en train de dire " just your voice" [juste ta voix], parole issue d'une chanson qu'Ursula, la sorcière de la mer, chante à la sirène Ariel. La famille a interprété cela comme un signe qu'Owen cherchait à retrouver sa voix. À partir de là, les dialogues de Disney sont devenus la langue que la famille Suskind a utilisée pour communiquer avec Owen, littéralement, se parlant les uns aux autres à travers la voix des différents personnages pour exprimer les questions de la vie de tous les jours. (Rosin, 2014, s/p; la traduction est la mienne)

Un autre compte-rendu du développement des usages d'Owen du dialogue Disney met bien en valeur les moments de nouveauté, de rupture, qui scandent ces usages répétitifs de la machine, qui nont rien de machinique.

Un jour, pour la fête d'anniversaire des neuf ans de son frère Walter, Owen se mit à pleurer. "Walter ne veut pas grandir, comme Mowgli ou Peter Pan", a dit Owen. Comparer son frère à des personnages de Disney était la chose la plus sophistiquée qu’Owen, alors âgé de six ans, ait prononcé depuis des années. (Harpaz, 2014, s/p)

Le recours à la machine pour donner lieu à la fonction de la lettre dans sa dimension d'inscription répétitive peut être réduite à une approche comportementaliste. C'est celle des chercheurs de Yale qui tentent détablir une thérapie standardisée à partir de l'Affinity Therapy. Ils décrivent ce qui est pour eux un «jeu de rôle» dont il faut encourager l'usage par un système de récompense.

En regard de cette raison comportementale, prenons le point de vue du thérapeute Dan Griffin, qui a longtemps travaillé avec Owen. Il isole, dans un entretien qu'il a eu avec Ron Suskind et une journaliste, Hanna Rosin, elle aussi mère d'un enfant autiste, le moment où Owen a pu faire un usage nouveau des dialogues Disney, dans une perspective psychodynamique: 
Dan Griffin : Je m’en souviens jusqu'à ce jour. Nous avions fait une pause au cours de la séance. En général Owen se sauvait de la pièce à toute vitesse et ce jour-là il est resté. Et vous avez joué une scène avec Iago [un personnage d'Aladdin] je crois. Et soudain il y eut cette décharge ionique dans la pièce. Tu étais beaucoup plus engagé. Il était beaucoup plus engagé. On avait l'impression que tout était possible. Il y avait une pure connexion et une pure joie, et ça a fait tilt, nous devons être capables d'exploiter ça ! Ron : Je vais te dire ce que ceétait. C’était la scène dans laquelle Iago dit, "OK, donc tu épouses la princesse et tu deviens le mari idiot". Owen l'a fait. Et j’ai répondu, comme Jafar, "J'aime la manière dont ta mauvais petite tête fonctionne" (Rosin, 2014, s/p)

Ce que Dan Griffin voit comme une décharge d'énergie présente dans la pièce me paraît mieux décrire ce point où la récupération de la voix permet de pousser au-delà du point où Owen supportait léchange, pour agrandir son circuit d'échange. Ce point de poussée au-delà est bien décrit par sa mère:

Tous ces enfants ont des obsessions et des affinités. La différence est que nous avons utilisé son affinité comme un outil, non seulement pour entrer en contact avec lui, en chantant des chansons, en regardant des films ensemble, en jouant avec des personnages et en les incarnant, mais aussi en poussant plus loin le processus et en commençant à l'utiliser pour l'aider dans ses études et dans ses relations sociales croissantes. (Harpaz, 2014, s/p)

Et Ron décrit aussi très bien l'envers de ces moments dénergie, la façon dont Owen a apprivoisé lénorme charge émotive des dessins animés en les traitant par la pure répétition:

Ces films se basent sur des thèmes puissants, comme ceux des frères Grimm et de mythes millénaires. Mais cest seulement depuis l’arrivée dans les foyers du magnétoscope que les enfants comme Owen peuvent rembobiner et systématiser l'apprentissage à leur propre rythme, et obtenir à partir de ces récits riches en émotions des choses qu'ils ne pouvaient pas obtenir des interactions humaines. Owen a utilisé les films pour comprendre qui il était et sa place dans le monde. Il se retrouvait dans la figure du personnage secondaire ou du compagnon [sidekick]. "Un personnage secondaire aide le héros à accomplir son destin." Cest une idée très forte. Il donnait des identités de personnages à d'autres enfants à lécole et disait: "Je suis le protecteur des personnages secondaires, aucun d'entre eux ne sera laissé pour compte." (Harpaz, 2014, s/p)

Aidé par les génériques Disney, Owen apprend à lire. Il aiguise ses compétences décriture en retravaillant les scénarios de classiques Disney. En parlant à travers les voix de personnages secondaires Disney, sages ou protecteurs, comme Rafiki, singe mandrill second-rôle dans Le Roi Lion, il trouve son chemin à travers les 
histoires mises en place par son thérapeute pour aborder des sentiments tels qu' “être perdu ou désorienté, être trompé, être frustré, ou perdre un ami." (Warner, 2014, s/p)

Puis Owen a pu être scolarisé à domicile, et enfin, dans une école spécialisée pour enfants autistes.

\section{Les variétés de la lettre et le traitement de la voix}

Nous voyons comment l'approche de l'Affinity Therapy permet de faire varier l'instance répétitive de la lettre. De même, l’œuvre de Daniel Tammet témoigne spécialement d'un amour de la matérialité de la lettre à travers les nombres, la trace, l'écriture, les mots, la voix poétique. Il sait émouvoir à partir d’effets métaphoriques rapprochant de façon inattendue des domaines séparés. Il fait de la métaphore le fondement de l'activité mathématique.

Ces registres, ces facultés que sont chanter, parler, écrire, dessiner, écouter de la musique, en faire, chacun se les approprie à sa façon et de manière hétérogène en tant quécriture, chiffre, fixation de la parole, images discontinues, ou par l'utilisation de la musique et de la mélodie. Des enfants totalement mutiques peuvent écrire énormément de choses, certaines illisibles, d'autres lisibles. D’autres enfants ne se situent ni du côté du parlé ni du côté de l'écrit mais chantent. D’autres encore comptent uniquement. Un sujet peut ainsi affirmer : «’ai tout oublié je sais juste que je sais compter». Les différents objets numériques permettent de mettre en valeur l'articulation de ces différents registres et ce qu'ils ont en commun : la possibilité de la répétition. Les claviers nous permettent de surmonter les difficultés de la motricité fine, qui suppose un rapport au corps et à son image particulièrement efficace dont tous les sujets ne peuvent pas bénéficier.

Dans la connexion du sujet autiste à l'autre, on sait le rôle des ordinateurs, aussi bien dans la procédure utilisée par B. Sellin que dans le dispositif de communication facilitée inventé par Rosemary Crossley ou dans la communication assistée utilisée par des collègues argentins. Le livre de Jean-Claude Maleval, L' autiste, son double et ses objets, a rendu compte des usages possibles des machines pour nouer un lien avec le sujet. Ron Suskind a proposé aux fabricants de logiciels de reconnaissance de voix SRI (qui ont donné leur nom à Siri) de permettre à ces assistants de choisir des voix personnalisées de dessins animés. Pour un enfant qui parle spécialement avec Aladdin, la voix de ce personnage pourrait être celle de l'assistant, permettant ainsi de diffracter les voix.

Dans cette répétition, s'agit-il de jeux de rôles ou d'un traitement de la voix de l'Autre? La question se pose, et ce dès les phénomènes décholalie dont on a pu montrer la place et la fonction active. La question se pose aussi à partir d’autres exemples que celui d'O. Suskind, comme celui de Tom (Junius, 2015) qui, arrivant à sept ans dans une école spécialisée, ne supporte pas la classe. Il joue seul, avec des 
bouts de phrases de dessin animé. Des phrases décontextualisées, hors sens, le traversent. Les parents s'inquiètent de le voir obnubilé par le dessin animé, par certaines phrases et chansons. Les premières rencontres avec Tom sont éclatées, il ouvre des boîtes, jette des objets à terre, s'empare des lunettes, allume/ éteint la lumière, se fixe sur un halogène muni de deux lampes : Tom utilise l'une pour regarder son reflet et, de l'autre, il fait un micro. Il chante et demande à la thérapeute de chanter avec lui. Dans cet éclatement des circuits de la voix et du regard qui ne sont pas mis en forme par une image stable du corps, Tom introduit peu à peu un rythme. "Il frappe le radiateur, et tout son corps se met en mouvement. Il se jette à terre, se cache sous la table, mime " $M$. Totem" en prenant un coffre dont il fait claquer le couvercle comme une bouche hurlante» (Junius, 2015, pp. 16-17). Il utilise aussi des phrases rituelles qui lui permettent de mieux supporter la séparation. "Par exemple, il dit "merci, merci, au revoir" aux objets, trop vivants, qui ont servi lors de la séance, et "merci, merci Justine". Je lui réponds "merci, merci Tom", et nous nous serrons la main » (Junius, 2015, pp. 16-17).

Au cours du développement des séances, là où était le cri et la bouche hurlante, viennent des bribes d'histoires aux allures catastrophiques.

Par exemple, il crie "Ils arrivent, allez, on se dépêche, on se dépêche ; maman ; mon enfant ; tous à la fourmilière !" [... J J'interviens : "Puis-je t'aider Tom?" Lui : "Non...Oui, regarde: quelqu'un est coincé dans l'immeuble, ouvre la fenêtre pour sauver Flash McQueen et les autres voitures." [...] Les phrases que Tom répète, tord et retord, [...] deviennent plus cohérentes. D’autre part, il intègre des oppositions signifiantes apprises en classe, telles que "gauche-droite", "jour-nuit", "chaud-froid". Lannée suivante [...] Tom supportant mieux dêtre en classe [...] participe à un atelier chant qui aboutit en fin d’année à un spectacle où il séduit la salle par sa performance. Il questionne le langage, pas seulement avec les dessins-animés et les chansons, mais à partir des énoncés entendus à lécole. (Junius, 2015, pp. 16-17)

Il peut s'inclure avec les autres. Sa façon de s'inclure est toujours reliée à son angoisse d'exclusion. «Par exemple, il se met devant l'éducateur adulte, et lui dit "Tu es viré !", puis il revient, va s'excuser...» (Junius, 2015, pp. 16-17). Avec une institutrice, «il inverse les rôles en lui demandant non pas de chanter, mais de compter» (Junius, 2015, pp. 16-17). C'est ainsi qu'il rentrera dans le système d'apprentissage des nombres et du comptage.

En trouvant une accroche dans la voix et le corps de la thérapeute, Tom a pu mettre à distance l'explosion qui le menaçait dans le chaos équivoque des différents registres pulsionnels de jouissance qui peu à peu vont sorganiser dans léchange, et se transformer en une élaboration qui va ensuite permettre un usage conversationnel du langage. 


\section{Variétés de la lettre et traitement du regard}

Après le traitement de la voix, venons-en au traitement du regard de l'Autre par les remaniements de l'espace de la vision, en particulier par le maniement du passage des représentations de deux à trois dimensions. Ces passages supposent la circulation du sujet dans un espace topologique déformable. Le sujet affecté de la rencontre avec une langue, qu’elle soit parlée, écrite ou numérique, est plongé dans un espace topologique qui nest pas celui de la géométrie euclidienne, qui nest pas celui du miroir plan. C'est un espace qui ne se raccorde pas facilement avec le corps. Les efforts des sujets pour les faire se rejoindre en attestent l'importance. Nous pouvons là encore partir de l'Affinity Therapy, puisque le dessin animé est d'abord saisi en 2D dans un espace plan. L’enfant raccorde son corps à lécran sur lequel se projettent les images de différentes façons. Il peut se coller à lécran télévisuel, ou au contraire s’en détourner, à loccasion lui tourner le dos.

Le rapport du champ de la vision dans l'espace plan se fait de multiples façons. Un autre exemple le montre. Un garçon avec autisme, Jules, a une passion pour chercher

(...) sur Google Earth et Google Maps à visualiser les lieux où il a vécu ainsi que son domicile actuel. [...] [Il] fait également des recherches sur les écrans comme tels : les écrans à cristaux liquides, la taille des écrans, les écrans tactiles, les images décrans écrasés. Et puis, il y a les recherches sur la 3D : les imprimantes 3D, les téléviseurs 3D, les tablettes 3D, les films 3D. "J'aime bien la 3D parce qu'on ne voit pas le bout "». (Louis, 2015, pp. 21-22)

Son intérêt pour la 3D se décline aussi "autour du chiffre 3 : la voiture Citroën C3, le triangle" (Louis, 2015, pp. 21-22). Il dit à l'éducateur:

\footnotetext{
"Toi, tu as une tête en triangle" [...] Il y a aussi "les biberons triangles" qui l'ont accompagné jusquà ses huit ans. "La 3D, c'est son biberon triangle, son obsession du moment", dira son père. Toutes ses recherches sur ordinateur se terminent presque invariablement par un coup de poing de Jules sur l'écran. "Boum, boum. C'est quand que je peux casser l'ordinateur? Pourquoi on ne peut pas casser? C'est interdit? C'est une petite ou une grosse bêtise?" (Louis, 2015, pp. 21-22)
}

Pour Jules, la tentative d'inclure son corps dans lécran ne consiste pas à s'y coller, mais à traverser le miroir. Le coup de poing final vise à s'inclure dans le champ de sa vision. Il ne cesse aussi de proposer:

(...) des constructions en carton auxquelles il assigne systématiquement le qualificatif "3D". Pour chaque réalisation, Jules s'affaire de longs moments sur les tracés de plans qu'il compile dans un cahier. Il trace méticuleusement ses modèles selon différentes perspectives, vue de haut, de face, vue latérale. [...] Il met en scène $[\ldots]$ des camions monstres écrasant de petits véhicules venant se fracasser sur les murs - dessiner des routes, des circuits vient border l'éclatement. (Louis, 2015, pp. 21-22). 
Nous voyons là aussi dans lécrasement sur les murs un mode de mise à plat de la représentation de lobjet et du corps:

Il se préoccupe davantage de choisir les couleurs, les papiers pour habiller ses créations. [...] Il seest lancé dans des décors qu'il a nommés "les décors vaches", il s'agit d'impressions avec les tâches caractéristiques [du pelage] des vaches. Il faut dire que le lait de vache l'anime beaucoup dans le quotidien. Les parents de Jules ayant constaté qu'il était plus agité à la fin des repas ont mis cela en lien avec sa consommation de produits laitiers qu'ils ont dès lors supprimés de son alimentation. Depuis, Jules n'a de cesse d'ouvrir les frigos de l'institution pour se saisir des briques de lait qu'il écrase. (Louis, 2015, pp. 21-22)

Latelier des «décors vaches» a permis qu'il ne passe pas son temps à ouvrir le frigidaire. La déformation de l'espace lui sert aussi à s’orienter dans les équivoques du langage. Pendant tout un temps, il était très intrigué par l'équivoque de «privé», entre «c'est réservé à lui» et c'est "privé de». Il élabore pendant cette période un objethabitacle particulier, «un hélicoptère bicolore. Il colle les deux faces de l'hélicoptère en disant "on va l'appeler un hélicoptère privé 3D" pour ponctuer sa recherche» (Louis, 2015, pp. 21-22). Le logicien Austin a écrit un beau livre dont le titre était Comment faire des choses avec des mots (Austin, 1962). Ici c'est l'inverse : il s'agit de faire des mots, de leur donner un sens «privé», en fabriquant des choses. C'est à partir des choses que Jules a fabriqué un nouvel usage conversationnel des mots.

Cette fabrique des corps et des mots comporte aussi bien des raccords d'images empruntées dans le bric-à-brac des ressources des discours disponibles, qui incluent toujours plus d'images. Elle nous permet de comprendre comment le sujet peut se déplacer dans le monde et l'interroger à partir d'un usage privé de la langue commune.

\section{La répétition, lalangue, l'écrit}

Ces modes de traitement des circuits de la voix et du regard supposent la rencontre du sujet avec autisme avec une lalangue dans le bain de langage où il est plongé. Et cette rencontre est très particulière. Elle passe peu par le babil : les études convergent pour établir sa pauvreté et son manque d'orientation sociale (Maleval, communication privée). L'absence de babil, l'absence d'équivoque langagière incarnée est présente dès le début des études sur l’autisme. Il y a par contre répétition de quelques mots/choses. Finalement, on constate la présence du signifiant pur. Le signifiant comme tel est bête. Il se présente ainsi, c'est un pur chiffrage et il chiffre tout. De plus, ce chiffrage est auto-érotique. Il y a pour l'enfant une jouissance particulière à chiffrer ainsi, qui n'est pas du tout centrée sur l'échange avec l'autre. Ce signifiant pur ne s'adresse pas. J.-C. Maleval souligne ce point:

Pendant les premiers mois, certains enfants autistes ne vocalisent pas, mais pleurent ou hurlent quand ils sont éveillés. D’autres restent silencieux. Quand le 
babil existe, il apparaît le plus souvent monotone (comparable à celui d'un bébé tombant de sommeil), sans entrain, sans inflexion intentionnelle. De surcroît il ne s'adapte pas aux productions maternelles. Certains autistes babillent dans la solitude, mais s'interrompent en présence d'un parent. (Maleval, communication privée).

Ce mode de surgissement de lalangue ne s'adresse pas, mais il laisse des traces sur le corps, il l'affecte. Ce mode d'affectation traduit l'inscription de la trace de jouissance sur la surface du corps, par le mode de répétition même. Si les enfants autistes ne s'articulent pas à la fonction de la parole, ils ne sont pas pour autant insensibles à l'instance de la lettre, puisque des sujets autistes qui présentent un retard dans l'acquisition du langage parlé disposent de l'écrit. B. Sellin écrit à un autre autiste, « je désire simplement comme toi investir mes instruments buccaux dans le langage. Mais j'erre encore très loin du langage » (Sellin, 1998, p. 130).

L'expression brutale de B. Sellin renvoie à un point que Lacan met en valeur dès le début de son enseignement. L'Autre et la voix ne s'articulent à aucun sensorium particulier, mais bien davantage au corps comme tel. Dans le Séminaire X, L'angoisse, en reprenant son Séminaire I, Lacan énonce: «il y a d'autres voies que vocales pour recevoir le langage. Le langage n'est pas vocalisation. Voyez les sourds » (Lacan, 2004, p. 317). Il avait déjà utilisé l'exemple des sourds dans son Séminaire III sur Les Psychoses:

C'est encore plus simple si nous pensons au sourd-muet, qui est susceptible de recevoir un discours par des signes visuels donnés au moyen des doigts [...] Je dirais plus - ce qu'il enregistre, à savoir la succession de ces signes, leur opposition sans laquelle il n'y a pas de succession, peut-on dire quà proprement parler il le voit? (Lacan, 1981, p. 154).

De même que dans le premier Séminaire où, grâce à saint Augustin, il avait coupé le lien entre le sensorium de la voix et la question de l'échange des signes, il le coupe ici avec la vision. Il ne considère pas, finalement, que la chaîne signifiante des signes « se voit» à proprement parler. Ça se perçoit au-delà de tout sensorium. On pourrait dire que c'est le corps dans toute sa surface qui est mobilisé. Le langage y fait impact au-delà des sensorium concernés. Ce qui se perçoit au-delà de tout sensorium, c'est l'opposition, dit le premier Lacan. On pourrait compléter ceci avec le dernier Lacan, en disant qu'il s'agit de la répétition comme telle.

Cette direction de recherche rejoint Laurent Mottron, chercheur canadien qui rejette la psychanalyse mais qui dit des choses sensées sur la question de l'autisme: 
Alors qu'il est très en retard pour le langage oral, il est très en avance pour le code écrit. Cet intérêt se fait hors de toute intention communicative, comme c'est le cas de lécholalie différée à son début. [...] Le langage écrit permet un abord non social du langage. (Mottron, 2016, cité par J. C Maleval, communication privée).

Le langage n'est pas fait pour communiquer. Le langage inscrit des expériences de jouissances, répétitives. Les traces de l’impact du langage sur le corps, puisqu'elles ne peuvent être connectées à la phone, sont inscrites sur le corps en des lieux étranges, qui définissent une topologie particulière de la voix. La voix peut être à l'intérieur du corps, vécu aussi comme un extérieur, un hors-corps, qui peut être imaginairement localisé dans un organe externe, une «voix artificielle». Instruments de musique, micros, marionnettes, doubles et objets divers peuvent être convoqués à cette fin, comme nous l'avons vu.

\section{La répétition et le langage privé}

Le refus de faire usage de la langue commune parlée et le rapport privilégié à lécrit s’accompagne volontiers de l'existence d'un langage privé, comme «élucubration de savoir» sur la langue commune. Il nest pas fait pour communiquer. Valérie GayCorajoud a noté que vers trois ans et demi, son fils Théo inventa une langue néologique que son entourage parvint à comprendre. Ses proches se mirent alors à lui parler en cette langue. Or, note sa mère, il refusa «que nous utilisions son langage en retour. Nous devions continuer de lui parler comme d'habitude et il nous répondait avec son langage réinventé» (Gay-Corajoud, 2018, p. 95). Ce langage essaie de se passer des équivoques de lalangue commune. C’est une tentative d'assigner des désignations de façon aussi univoques que possible.

L'exemple de la langue inventée par $\mathrm{D}$. Tammet me paraît répondre à la même volonté d'extraction d'un langage univoque à partir de l'impact de la lalangue sur le corps. Dès sa petite enfance, il adopte le langage des nombres, mais les nombres ont un impact étrange sur son corps. Il possède une perception synesthésique de chacun de ceux-ci, de sorte qu'ils lui apparaissent «comme autant de formes, de couleurs, de textures et de mouvements. Le nombre 1, par exemple, [écrit-il] est d'un blanc brillant et éclatant, comme quelqu'un qui dirige le faisceau d'une lampe torche directement dans mes yeux. » (Tammet, 2009, p. 11). Chaque élément-nombre aboutit à un langage par cette combinaison inédite de forme et démotion qui fait du nombre un objet complexe, équivoque du point de vue de la référence. Comme dans le cas du Un, où le nombre se trouve lié à l'intrusion de la lampe torche qui vise les yeux.

Cette langue privée est chargée d'une jouissance exceptionnelle et lui procure d'intenses émotions. «Cette dimension esthétique de ma synesthésie a de bons et de mauvais côtés» (Tammet, 2009, p. 15) entre «frisson d'excitation et de plaisir» et «m'irrite et me met mal à l'aise». Ce ne sont pas exactement des affects, mais des événements de corps qui se produisent. 
La conférence à l'Espace des sciences de Rennes en 2018 (Tammet, 2018) nous a permis d'entendre, en réponse à la demande d'une spectatrice, $\mathrm{D}$. Tammet réciter un poème de nombres qu'il a composé. Lémotion pure est patente, mais elle reste obscure aux auditeurs - comme il le notait lui-même. Quand il était adolescent, ça ne passait pas. Cette langue maternelle des nombres reste opaque. Elle n'est pas branchée sur l'autre, tout en étant le dépôt d’expériences d’émotions vives.

Avec son autre langue privée, le Mänti, on rencontre la même intention déliminer l'équivoque, le discordant de la langue commune pour trouver un référent précis. Le Mänti permettait de «trouver des mots pour exprimer mes expériences particulières. Parfois, quand je ressentais une émotion particulièrement forte, ou que je faisais l'expérience de quelque chose d'extrêmement beau, un nouveau mot se formait spontanément dans mon esprit pour l'exprimer, et je ne savais pas d'où il pouvait bien venir. Au contraire, jai souvent trouvé la langue de mes camarades déroutante et discordante " (Tammet, 2009, p. 216). Cette opération originale de nomination s'est révélée pour $\mathrm{D}$. Tammet aussi efficace que ce que visent à obtenir les lunettes Google pour aider les sujets autistes à nommer des émotions, en couplant logiciels de reconnaissance de visages et étiquettes démotions. Elle révèle aussi que cet étiquetage involontaire est fait par la machine fondamentale en nous quest le langage, dont on ne sait d'où il vient.

Ce langage privé se veut exact : «J'ai continué de rêver au jour où je parlerais une langue bien à moi...et que cela exprimerait exactement quelque chose de moimême» (Tammet, 2009, p. 216). D’autre part, c'est une élucubration de savoir : «J'essaye d'inventer en Mänti des mots qui établissent d’autres liens entre les choses : hamma (dent) et hemme (fourmi-un insecte qui mord), rât (fil électrique) et ratio (radio) par exemple» (Tammet, 2009, p. 217). Lors de cette conférence, D. Tammet notait qu'il n’avait plus besoin de passer par sa langue inventée maintenant qu'il était devenu écrivain et qu'il avait forgé sa langue propre dans ses livres. En ce sens, loin dêtre une «barrière autistique de plus à la communication», comme certains l'avancent, le langage privé fait plutôt bord transitoire, jusqu'à ce qu'il perde sa spécificité. Karim Nazeer rend compte de la curieuse facilité d'apprendre des langues réputées complexes par le fait que «dans celles-ci, il y a tant de règles et de structures que cela élimine les équivoques qui posent problème aux autistes. «Un sens/un mot serait pour eux l'idéal» (Nazeer, 2007, p. 26).

On peut opposer à la construction d'une langue privée le surgissement des vocalisations involontaires, comme celles qui traversent Temple Grandin à l'occasion d'un accident de voiture advenu dans sa petite enfance quand elle était encore mutique:

Maman a essayé de tourner le volant, se souvient-elle, mais il était trop tard. J’ai entendu s'écraser le métal et j’ai senti le choc violent... J'ai crié "glace, glace" pendant que le verre cassé pleuvait partout sur moi. Je n'avais pas du tout peur. C’était assez passionnant. (Grandin, 1997, p. 39) 
Au moment de la menace la plus grande, surgit une vocalisation qui désigne la glace qui se brise. «Quelquefois, je prononçais des mots, comme je l'avais fait pour glace, très clairement. Cela se produisait lors des moments de grande tension comme l'accident de voiture, quand le stress arrivait à vaincre la barrière, qui d'habitude m’empêchait de parler» (Grandin, 1997, p. 39). L'originalité de cette émotion est celle d'un stress, mais sans peur ressentie. Que le mot qui surgisse ait rapport avec le miroir qui se brise n'est peut-être pas sans intérêt.

Il y a aussi la première phrase prononcée par B. Sellin: «rends-moi ma boule», adressée à son père qui venait de lui prendre l'un de ses objets autistiques (Sellin, 1994, p. 24). S'agit-il d'une "parole automatique», ou du témoignage d'une impossibilité d'entrer dans le langage par la voie de la parole, mais qui se situe sur les franges d'un phénomène entre lettre et parole? Dans le dernier enseignement de Lacan, ces phénomènes de franges sont appelés «jaculations». Dans la série des «jaculations», il essaie d'inscrire l'interprétation psychanalytique du côté de quelque chose qui est au-delà de la parole, un mélange de la parole et de lécrit. Dans cet audelà de la parole, Lacan met aussi bien les jaculations, les cris du maître Zen que les effets émouvants obtenus par la poésie.

Dans ces différents champs, depuis le babil jusquà la vocalisation éruptive, en passant par l'invention d'un langage privé, cet agencement témoigne d'une subjectivité autistique qui se produit dans la construction du langage privé comme étant le témoignage par excellence de la construction d'un bord spécifique entre le sujet et l'Autre auquel il a affaire. Ces phénomènes originaux témoignent de la rencontre avec le bain de lalangue dans lequel le sujet est plongé, dans toutes ses variétés. Le sujet s'extrait de lalangue commune en prenant appui sur la répétition de l'inscription des mots sur le corps. Le sujet n'est pas fait pour communiquer, mais pour s'insérer dans le monde de façon autoérotique. Et ce sont les circuits que le sujet composera qui lui permettront d’obtenir cette inclusion dans le monde. La cure de Louange, l'enfant du placard, commentée par J.-C. Maleval et Michel Grollier, en témoigne remarquablement (Maleval et Grollier, 2019). Par les voies de la création, l’euvre de D. Tammet est un exemple, maintenant connu de tous, de la construction d'une langue particulière, longuement élaborée, par un processus qui l'a rendu sensible à ce qui échappera à la machine et au «Test de Turing».

\section{Notes}

${ }^{1}$ La présentation de ce livre a eu lieu, pour la France, à l'université Rennes 2 lors du colloque international «Affinity Therapy : un nouveau regard sur l'autisme», les 5 et 6 mars 2015. R. Suskind et D. Griffin sont les inventeurs du terme Affinity Therapy. 


\section{Références}

Austin, J. L. (1962). How to do things with words. Harvard University Press.

Chérel, M. (2019). Argument du Colloque «Autisme: Numérique et Robotique. Quel partenaire privilégié au 21e siècle?». Université Rennes 2, 7 et 8 novembre 2019. HTTPS://WWW.LAIREDU.FR/ COLLECTION/COLLOQUE-AUTISME-NUMERIQUEET-ROBOTIQUE-QUEL-PARTENAIRE-PRIVILEGIEAU-21E-SIECLE/

Gay-Corajoud, V. (2018). Nos mondes entremêlés. Imprim'vert.

Grandin, T. (1997). Ma vie d'autiste. Odile Jacob.

Harpaz, B. J. (2014). A little movie magic: How Disney films unlocked an autistic boy's emotions. Entretien avec R. et C. Suskind, 26 mars 2014. AZcentral. HTTPS://EU.AZCENTRAL.COM/STORY/ ENTERTAINMENT/B OOKS/2014/03/26/DISNEYFILMS-AUTISTIC-BOY-EMOTIONS/6913067

Junius, J. (2015). Apprendre et se laisser apprendre. Quarto, 111, 16-17.

Lacan, J. (1981). Le séminaire. Livre III. Les psychoses (texte établi par J.-A Miller). Seuil.

Lacan, J. (2004). Le séminaire. Livre X. Langoisse (texte établi par J.-A Miller). Seuil.

Laurent, É. (2012). La bataille de l'autisme. Navarin/Le Champ Freudien.

Louis, S. (2015). Tentatives pour se faire partenaire. Quarto, 111, 21-22.

Maleval, J. C. (communication privée). De l'aliénation retenue chez lautiste. Communication privée, à paraître.

Maleval, J.-C. (2009). L'autiste, son double et ses objets. Presses Universitaires de Rennes.

Maleval, J.-C. y Grollier, M. (2019). Une démonstration éblouissante. Un enfant autiste en analyse. Lacan Quotidien, 831, 12 avril 2019.
HTTPS://WWW.LACANQUOTIDIEN.FR.

Metz, C. (2019). Google Glass for autistic children. The New York Times, 17 de julio de 2019.

Mottron, L. (2016). Lintervention précoce pour enfants autistes. Mardaga. [Cité par J. C. Maleval, comunicación personal]

Nazeer, K. (2007). Laissez entrer les idiots. Témoignage d'un autiste. Seuil.

Newman J. (2014). To Siri, with love. The New York Times, 17 octobre 2014.

Reliquet, S. y Reliquet, Ph. (2011). Écouter Haendel. Gallimard

Rosin, H. (2014). A pathway, not a prison. Entrevista con R. y C. Suskind. Slate, 1ro de abril de 2014. HTTPS://SLATE.COM/HUMAN-INTEREST/2014/04/ LIFE-ANIMATED-TALKING-ABOUT-AFFINITIESAU TISM - AND - DISNEY - WITH - RON - SUSKIND CORNELIA-SUSKIND-AND-DAN-GRIFFIN.HTML

Sellin, B. (1994). Une âme prisonnière. Robert Laffont.

Sellin, B. (1998). La solitude du déserteur. Robert Laffont.

Suskind, R. (2016). Life, animated: A story of sidekicks, heroes, and autism. Hyperion.

Tammet, D. (2009). Je suis né un jour bleu. J’Ai Lu.

Tammet, D. (2018). Chaque mot est un oiseau à qui l'on apprend à chanter. Conférence, Espace des sciences, Rennes, 16 janvier 2018.

Warner, J. (2014). A family's hard journey, with Disney as a guide. The New York Times, 21 mars 2014. HTTPS://WWW.NYTIMES.COM/2014/05/22/BOOKS/ LIFE-ANIMATED-BY-RON-SUSKIND.HTML 